Efficacy of single and double doses of albendazole and mebendazole alone and in combination in the treatment of Trichuris trichiura in school-age children in Uganda

Namwanje, Harriet; Kabatereine, Narcis; Olsen, Annette

Publication date:

2010

Document version

Peer reviewed version

Citation for published version (APA):

Namwanje, H., Kabatereine, N., \& Olsen, A. (2010). Efficacy of single and double doses of albendazole and mebendazole alone and in combination in the treatment of Trichuris trichiura in school-age children in Uganda. Poster session presented at ICOPA XII. The XIIth International Congress of Parasitology, Melbourne, Australia. 


\section{Efficacy of single and double doses of albendazole and mebendazole alone and in combination in the treatment of Trichuris trichiura in school-age children in Uganda.}

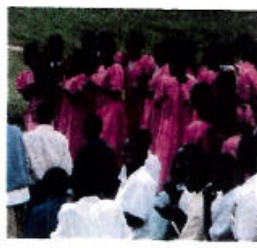

Introduction

Infections with Trichuris trichiura are estimated to affect approximately 1 billion people globally with high prevalences and intensities in pre-school and school-age children. Albendazole (ALB) and mebendazole (MBD) are recommended drugs for controlling this infection, but unfortunately cure and egg reduction rates are low.

$A L B$ and $M B D$ are both benzimidazoles, but have quite different chemical structures. Thus, a combination of ALB and MBD may act synergistically compared to the actions of the single drugs ALB and $\mathrm{MBD}$ in comparable doses.

A randomised clinical trial was conducted in Kabale district, south western Uganda to compare the efficacy of a single and a double dose of a combination of ALB $(400 \mathrm{mg})$ and MBD $(500 \mathrm{mg})$ with single and double doses of each drug given alone in the treatment of T. trichiura.

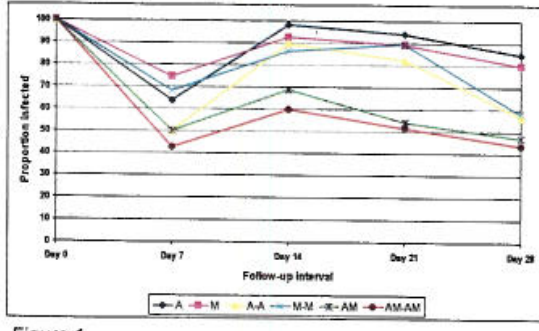

Figure 1

Results and discussion

The proportion of infected children (Figure 1) was significantly lower in the two combination groups (both single and double doses) than in all other treatment groups $(P<0.001)$ on day 14 and 21 after treatment. The number of egg excreted (geometric mean intensity of positives, Figure 2) was significantly lower in the two combination groups compared to all other treatment groups $(P<0.001)$ from day 7 and onwards.

In conclusion, the combination of ALB and MBD (both doses) was better than a single or double dose of each drug given alone. One reason could be that the contact time in the host intestine between drug and parasite are prolonged because MBD is the active drug, while it is the first metabolite of ALB (ALB sulphoxide) which is antheiminthically potent. The decrease (day 0 to 7) and subsequently increase (from day 7 ) in egg excretion have been seen in earlier studies and may be due to a temporary reduction in the metabolism of the worm.

All the tested regimens of ALB and MBD have, however, low efficacy against $T$. trichiura in Uganda. 